

\title{
A complex mixed-mode crack propagation test performed with a 6-axis testing machine and full-field measurements propagation
}

A. Carpiuc-Prisacari, M. Poncelet, K. Kazymyrenko, Hugo Leclerc, François Hild

\section{To cite this version:}

A. Carpiuc-Prisacari, M. Poncelet, K. Kazymyrenko, Hugo Leclerc, François Hild. A complex mixedmode crack propagation test performed with a 6 -axis testing machine and full-field measurements propagation. Engineering Fracture Mechanics, 2017, 176, pp.1-22. 10.1016/j.engfracmech.2017.01.013 . hal-01480988

\section{HAL Id: hal-01480988 \\ https://hal.science/hal-01480988}

Submitted on 2 Mar 2017

HAL is a multi-disciplinary open access archive for the deposit and dissemination of scientific research documents, whether they are published or not. The documents may come from teaching and research institutions in France or abroad, or from public or private research centers.
L'archive ouverte pluridisciplinaire HAL, est destinée au dépôt et à la diffusion de documents scientifiques de niveau recherche, publiés ou non, émanant des établissements d'enseignement et de recherche français ou étrangers, des laboratoires publics ou privés. 


\title{
A complex mixed-mode crack propagation test performed with a 6-axis testing machine and full-field measurements
}

\author{
A. Carpiuc-Prisacari ${ }^{\mathrm{a}, \mathrm{b}, \mathrm{c}, *}$, M. Poncelet ${ }^{\mathrm{a}}$, K. Kazymyrenko $^{\mathrm{b}, \mathrm{c}}$, H. Leclerc $^{\mathrm{a}}$, F. \\ Hild $^{\text {a }}$ \\ ${ }^{a}$ LMT-Cachan/ENS-Cachan/CNRS/Université Paris Saclay \\ 61 avenue du Président Wilson, 94235 Cachan Cedex, France \\ ${ }^{b}$ Institut des Sciences de la Mécanique et Applications Industrielles, UMR \\ EDF-CNRS-CEA-ENSTA 9219 Université Paris-Saclay, 828 Boulevard des Maréchaux, \\ 91762 Palaiseau Cedex, France \\ ${ }^{c}$ EDF Lab Paris-Saclay, 7, boulevard Gaspard Monge, 91120 Palaiseau, France
}

\begin{abstract}
A new type of mixed mode crack propagation test is proposed. A single crack is initiated and propagates in a stable way up to complete failure. A combination of tensile, shear and in-plane rotation performed by a 6 -axis testing machine is prescribed. The rotation creates a tension/compression gradient in the sample ensuring the stability, while the shear direction is closely related to the orientation of the crack and the tensile load is responsible for the actual propagation. The experiment is performed in an interactive manner, namely, depending on the crack tip position estimated by Digital Image Correlation (DIC) during the test, the loading is changed to bifurcate the crack. The displacements of the sample surfaces are assessed using multiple DIC measurements and displacement sensors. The displacement fields on each face of the sample give access to the crack pattern, and also to the actual boundary conditions that are crucial for a faithful numerical analysis of the test. Last, the 6 load components are recorded enabling for a complete description of the 3D mechanical behavior of the specimen.
\end{abstract}

Keywords: Concrete, Digital Image Correlation, Mixed-mode crack

\footnotetext{
${ }^{*}$ A. Carpiuc-Prisacari

Email address: andreea.carpiuc@edf.fr (A. Carpiuc-Prisacari)
} 
propagation, Multiaxial testing

\section{Introduction}

The development of multiaxial testing machines is rather recent. They have the advantage of applying boundary conditions very close to real-life cases, but also various loading types on the same specimen geometry. Moreover even 5 tests requiring less loading axes (e.g., tensile test) may be performed without relying on the mechanical stiffness to ensure zero displacements along the other directions. Loading axes as well as 'constrained' axes are controlled by the regulation loop.

Parallel machines seem good candidates for material testing because of their high accuracy and stiffness in comparison with serial machines [1]. The most used one is the 6-axis Gough-Stewart platform (a.k.a. 'hexapod') architecture 2, 3], invented in the 1960s for tire testing, but only recently used for structure (e.g., spine stiffness measurement [4, 5, 6]) or material (i.e., crimped wool [7, carbon composite [8] or concrete [9]) testing.

In terms of measurement, the current trend is the spread of easy, rich and accurate full-field measurement techniques such as Digital Image Correlation (DIC) [10. The associated technological revolutions are the appearance of digital cameras and the increasing computation power. A large range of algorithms has then been implemented and improved to gain from these technological changes. From early 2D local approaches [11, 12] to Finite-Element descriptions [13, 14, and integrated approaches [15, the measurement techniques have been constantly improved and adapted to the studied problem. Stereovision has also been combined to DIC to measure 3D shapes and 3D surface displacements [16, 17, and more recently 3D volumetric displacement measurements [18 with the use of X-ray tomography. These new techniques totally change the experiment-simulation dialog since the boundary conditions can now be described with 2D or 3D full-field data, as well as the kinematic field in the region of interest of the specimen. 
These two breakthroughs combined together enable for the improvement of are the ones where Saint-Venant's assumption cannot be made since it is now possible to accurately control and measure Dirichlet boundary conditions.

The so-called Nooru-Mohamed (NM) test [19, 20] is one of them, initially designed to maximize the dependence of the results to the boundary condishear quasi-brittle cement-base specimens up to failure. The specimen shape, which is very compact, ensures that the mixed mode crack propagation highly depends on the boundary conditions. Two experimental challenges arose. First, due to the stiffness of the specimen, the difference of orders of magnitude be40 tween the size of the specimen (i.e., $200 \mathrm{~mm}$ ), the characteristic displacement at failure (i.e., $\approx 20 \mu \mathrm{m}$ ) and the applied load (i.e., $\approx 10 \mathrm{kN}$ ) makes trustworthy measurements complex. Second, as underlined by Nooru-Mohamed himself [22], only the displacements along the two loading axes could be acquired, while 3D displacements would be of interest because of the high sensitivity to boundary conditions. In spite of these drawbacks and the fact that only point-wise kinematic data were available (in addition to the final crack path), the so-called NM tests have become a benchmark for numerous models and numerical implementations [23, 24, 25, 26, 27, 28, 29, 30, 31, 32, 33, 34, 35] since it is one of the few mixed-mode crack propagation experiments (i.e., Refs. 36, 37, 38, 39] are among the few other mixed-mode crack propagation tests on cement-based material).

It is thus proposed to perform a new test inspired by Nooru-Mohamed's configuration. The specimen shape is very close but a single notch is preferred to a double notched sample. The nonproportional loading history is still composed 55 of a vertical translation, which is corresponding to a tensile loading, and a horizontal translation corresponding to global 'shear,' but also of a additional rotation inducing a gradient of tensile stresses. The loading is applied with a 6-axis testing machine. This new experimental protocol has several advantages. First, since several full-field kinematic measurement systems are used, even if 
the applied boundary conditions do not exactly match the theoretical ones, the results can still be used for simulation purposes. Second, the addition of the rotation leads to stable crack propagation for the majority of the test so that the quantity of acquired data is very important contrary to a test that becomes unstable sooner. Third, several crack reorientations with non-negligible straight propagations between each of them allows damage and fracture models to be challenged for both propagation velocity and reorientation criterion. Last, the zigzag crack path is a convenient way to validate branching models.

The paper is organized as follows. First the material and the specimen will be described in Section 2.1, then the testing machine (Section 2.2), and how the specimen is gripped to the machine (Section 2.3). The complete instrumentation of the test will be thoroughly presented in Section 2.5, in addition to the validation of the experimental setup (Section 2.6). Last, the results of the test will be discussed (section 3).

\section{Experimental protocol}

75 2.1. Specimen 6 material

The studied specimen is a $50 \times 200 \times 200 \mathrm{~mm}$ mortar parallelepiped, with one $5 \times 25 \mathrm{~mm}$ sawed notch (Figure 1). The point $O_{\text {sup }}^{\text {spe }}$ used hereafter is located in the middle of the upper surface, at the interface between the concrete specimen and the glue layer below the steel plates.

The considered material is a mortar equivalent to the concrete used for the Vercors mock-up [40, a 1:3 scale version of a nuclear confinement building. The mortar composition is given in Table 1. The specimen is cast vertically,i.e., the upper boundary condition corresponds to the free surface during casting. Even though the mortar is very fluid during casting, a tomographic measure85 ment (figure 2) reveals no heterogeneity of sand density in the region of crack propagation. The specimen is stored in a water basin with other specimens from the same casting for a temperature ranging from 20 to $26^{\circ} \mathrm{C}$. The specimen is 




Figure 1: Geometry of the specimen (dimensions in $\mathrm{mm}$ ). The dark grey parts are U-shaped steel plates.

taken out of the tank 6 days before the test so that the surface is dry to apply the speckle pattern for DIC purposes (see Sections 2.5 and 2.3.

Table 1: Concrete mix composition.

\begin{tabular}{|c|c|c|c|}
\hline Effective water $\left[\mathrm{kg} / \mathrm{m}^{3}\right]$ & Cement $\left[\mathrm{kg} / \mathrm{m}^{3}\right]$ & Sand $0 / 4\left[\mathrm{~kg} / \mathrm{m}^{3}\right]$ & Plasticizer $\left[\mathrm{kg} / \mathrm{m}^{3}\right]$ \\
\hline 319 & 611 & 1235 & 5.25 \\
\hline
\end{tabular}

The main mechanical properties of the mortar are obtained with a series of five 3 -point flexural and compression tests on $40 \times 40 \times 160 \mathrm{~mm}$ specimens from the same casting. The specimens were taken out of the water basin 3 days before the tests. The 3-point flexural tests follow the standard 196-1 NFEN [41] for 3 specimens. To check whether the measured Young's modulus was rate-independent, 2 other specimens were tested with a load rate 100 times lower, corresponding to the order of magnitude of the strain rate of the tests performed on the hexapod. There was no noticeable variation of tensile strength (Table 2), and only a slight increase of Young's modulus. The standard (1961 NF-EN) compression test was then performed on the 5 broken specimens, and the measured compression strength is equal to $80 \pm 2.89 \mathrm{MPa}$.

The fracture energy $G_{f}$ is measured with 3 -points bering tests following 




Figure 2: Tomographic image of the specimen before test. The density of sand is homogeneous. The darker upper part is due to tomographic reconstruction.

Table 2: Average ( \pm standard deviation) concrete properties obtained for two loading rates.

\begin{tabular}{|c|c|c|}
\hline Load rate & Young's modulus & Tensile strength \\
\hline $50.0 \mathrm{~N} / \mathrm{s}$ & $17.25 \pm 0.74 \mathrm{GPa}$ & $3.8 \pm 0.4 \mathrm{MPa}$ \\
\hline $00.5 \mathrm{~N} / \mathrm{s}$ & $20.03 \pm 0.05 \mathrm{GPa}$ & $4.1 \pm 0.4 \mathrm{MPa}$ \\
\hline
\end{tabular}


the RILEM 1985 standard [42] on 6 specimens. To assess the influence of the moisture, 3 specimens are tested 7 days after being taken out of the basin, and 3 specimens 12 hours after. No particular trend is found. The force/deflection curves are given Figure 3. The average $G_{f}$ value is $114.6 \pm 18.8 \mathrm{~N} / \mathrm{m}$.

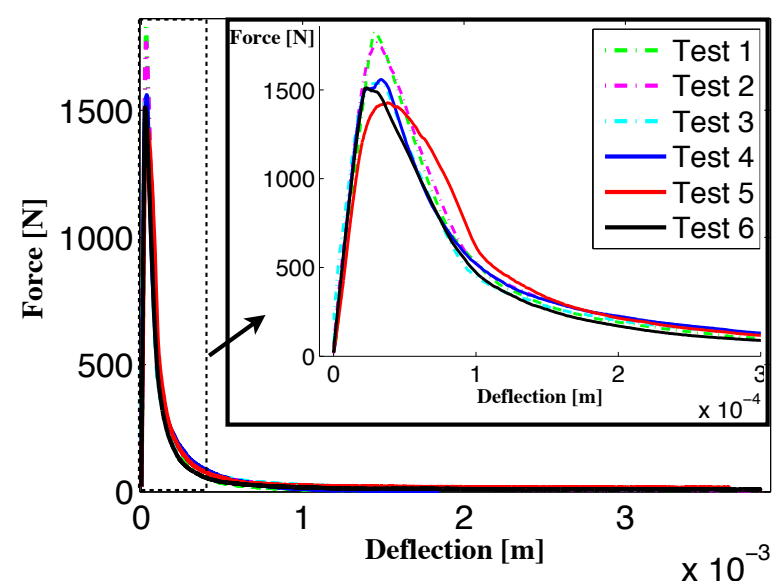

Figure 3: Force-deflection curves obtained during the 3-point bending tests. The first 3 specimens were out of the basin for 7 days, the last 3 for 12 hours.

\subsection{Testing machine}

\subsubsection{Machine hardware}

The test is performed on a 6-axis electromechanical testing machine 9], based on a Bosch-Rexoth hexapod robot (Figure 4). The load capacity depends on the position of the end-effector, but may be summarized as approximately $120 \mathrm{kN} \& 70 \mathrm{kNm}$ along $\underline{Z}$ direction, $50 \mathrm{kN} \& 45 \mathrm{kNm}$ in the $\underline{X}-\underline{Y}$ plane (the maxima cannot be reached in each direction at the same time because of the parallel architecture of the machine). The displacement space is also nontrivial but can be roughly represented as $\pm 250 \mathrm{~mm}$ and $\pm 22^{\circ}$ along $\underline{X}, \underline{Y}$ and $\underline{Z}$ directions. The theoretical actuator displacement resolution equals $0.15 \mu \mathrm{m}$, leading to translation errors less than $0.14 \mu \mathrm{m}$ (resp. $0.09 \mu \mathrm{m}, 0.08 \mu \mathrm{m}$ ) along $\underline{X}$ (resp. $\underline{Y}, \underline{Z}$ ) direction and rotation errors less than $0.15 \times 10^{-6} \mathrm{rad}$ when 
the machine is set in the middle of its displacement space (i.e., at mid-length of the actuators).

The end-effector (labeled (4) in Figure 4) is equipped with an octogonal reference plate oriented along the hexapod own coordinate system $\underline{X}, \underline{Y}, \underline{Z}$ with a tilt less than $1 \mu \mathrm{m}$ for $1 \mathrm{~mm}$ for each of the 3 directions. It enables fixing parts to be screwed on it as well as targets for Digital Image Correlation purposes. Several of its surfaces are ground to enable displacement measurement with contact sensors. A small passive hexapod (labeled (7) in Figure 4) is positioned under the large active one. Its legs are instrumented with strain gages and it is used as a 6-axis load cell. Its measurement uncertainties are $80 \mathrm{~N} \& 20 \mathrm{Nm}$.

\subsubsection{Machine control}

It is theoretically possible to control a parallel machine in force mode, or even in more complex modes such impedance control [43. The present machine is nonetheless only driven in a displacement mode because of its axis card. The signal of the load cell is thus only recorded but not used for control purposes.

Even if the intrinsic stiffness of parallel machines is high, performing a test with a joint position control is not sufficiently accurate for a cement-based specimen as described before. Different techniques have been developed to circumvent this issue. The first one is to precisely assess the machine stiffness and take into account the specimen own stiffness change in the control loop of the machine [44]. Another solution is to directly measure the position of the endeffector and use an end-effector position control. The latter can be obtained with a set of uniaxial sensors [4, 8, 6, or with a non-contact optical full-field measurement technique [45. One advantage of the optical method is that it does not hide the space around the specimen if used from a long distance. This last technique is chosen herein.

The control is done with a standard proportional integral (PI) corrector for each actuator as for a serial machine. The specificity is that the actuator length measurement is performed with a dedicated Digital Image Correlation (DIC) technique [45]. The principle consists of taking pictures of several flat targets 


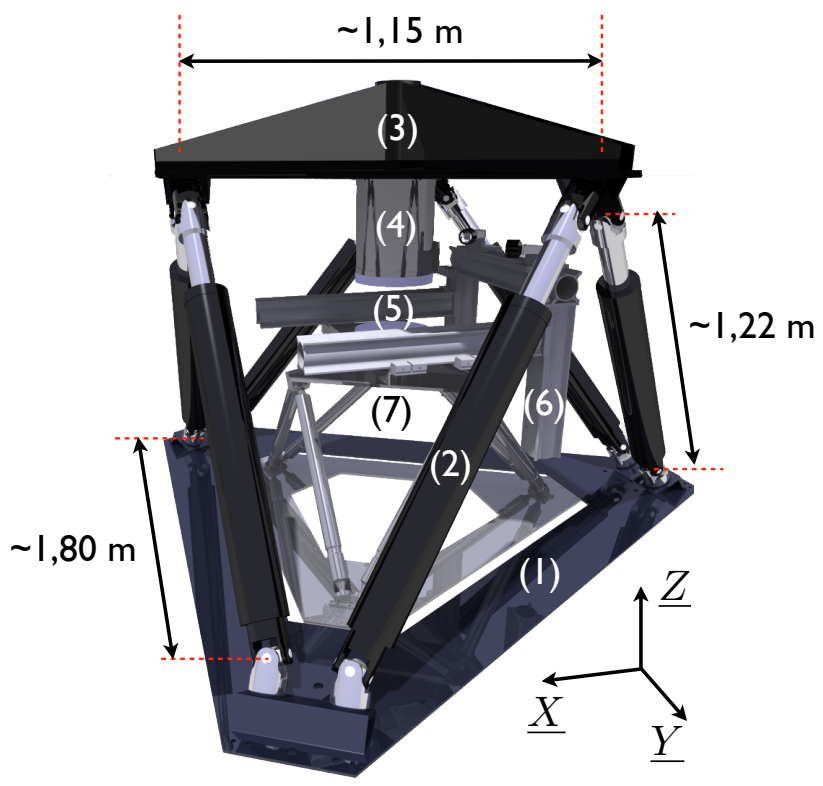

(a)

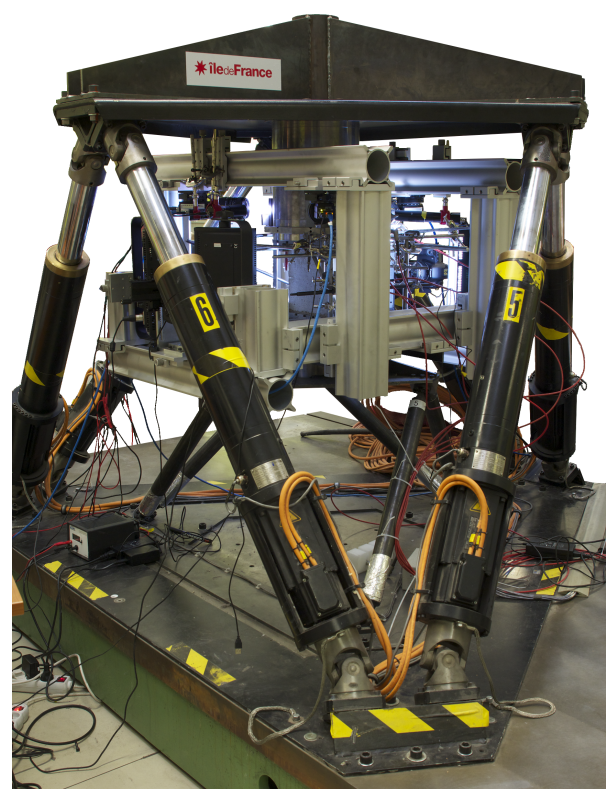

(b)

Figure 4: Six-axis testing machine. (a) CAD view with (1) base, (2) actuators, (3) moving platform, (4) upper end-effector, (5) room for specimen, (6) optical setup in close configuration with two cameras, (7) passive hexapod. The approximative lengths of 3 different parts are given for the sake of clarity. (b) Real testing machine fully equiped (apart from the 4 cameras for stereoDIC). 
mounted on the end-effector with several cameras mounted on the passive hexapod. The actuator lengths are sought as the solution to the minimization of a global problem, i.e., they minimize the difference between the current image and the reference image for each camera. The displacement of each target in front of its camera is described by 6 elementary rigid body motions. Because of the very small number of degrees of freedom, the DIC algorithm is integrated, namely, the measured degrees of freedom are directly the quantities of interest [46], which offers a reduced computation time. Moreover the algorithm is implemented on Graphics Processing Units (GPUs), which enables for the parallelization of the computation.

To relate the actuator lengths to the displacement observed by each camera, calibration matrices are used. These matrices are obtained via a calibration procedure where each actuator is shifted individually one after the other to record their influence on each target displacement. One pays close attention to move each actuator both ways to delete any clearance during the calibration, and prior to it (i.e., before taking the reference images). The procedure is followed just before the test with the targets and cameras in place, but without the specimen mounted on the machine. Since the procedure takes few minutes, it is started about an hour after the actuators and the lights are on so that the thermal deviations due to warm-up will not induce errors in the calibration matrices.

In the present case, 3 targets covered with a black and white speckle and 3 cameras (Leutron Picsight P141M-GigE camera, definition: $1392 \times 1040$ pixels, 20 frames per second, 12-bit digitization) are used. The cameras are fitted with the same Zeiss lens (50 mm F1.4) and the same Kenko $(20+12 \mathrm{~mm})$ extension tubes. The calibration procedure is carried out with an elementary displacement equal to $100 \mu \mathrm{m}$. The standard uncertainty of the actuator length 175 measurement with this technique is sufficiently low to accurately control the hexapod machine (i.e., $\approx 0.4 \mu \mathrm{m}$ with the present optical setup). Moreover the frequency of measurement $(20 \mathrm{~Hz})$ is sufficient to perform a quasi-static test. It has been shown 45 that the displacement error of the machine in such a 
configuration is less than $2 \mu \mathrm{m}$, and can be reasonably assessed to less than $1 \mu \mathrm{m}$ because the error is partly due to the validation setup at this scale.

\subsection{Gripping the specimen 85 boundary conditions}

For the original NM tests and the present one, the loading is transmitted from the machine to the specimen through high performance glue (i.e., Sikadur 31 epoxy glue, cured during $24 \mathrm{~h}$ before test). Moreover, the loading was applied by L-shaped steel plates. In the present case where the setup is designed to perform shear in both directions, flat or U-shaped parts should be used. Numerical simulations with a nonlocal damage model [47] (see Appendix A) show that damage occurs in the vicinity of the glued interface for flat parts (example of a simulation performed by allpying a proportional tensile-shear loading to the sample, Figure 5(a)). Experimental results also show crack initiations and propagations along these interfaces. Since the measured displacements on the specimen with DIC and not the ones of the machine will be used, interface debonding is not a limitation for the simulations. However, the gradual debonding of the interface implies that the theoretical boundary conditions are not at all applied to the specimen. Consequently, reorienting the main crack is more difficult if not impossible. To prevent that effect, monolithic U-shaped parts (Figures 1 and $9(\mathrm{a})$ ) with 18.5 -mm wide $\times 25$-mm height sidewalls made of steel are used to maintain the specimen with a $1.5-\mathrm{mm}$ thick glue layer. Numerical simulations with perfectly rigid U-shaped boundary conditions still predict damage initiation at the tip of the sidewalls (Figure 5(b)) but this boundary effect vanishes on real tests because of the compliance of the glue and of the sidewalls.

\subsection{Loading path}

The loading path consists of a series of tension, rotation and shear steps ${ }_{205}$ (Figure 6(a)) that lead to initiation, propagation and reorientation of the crack following a zigzag path (Figure 6(b)). 


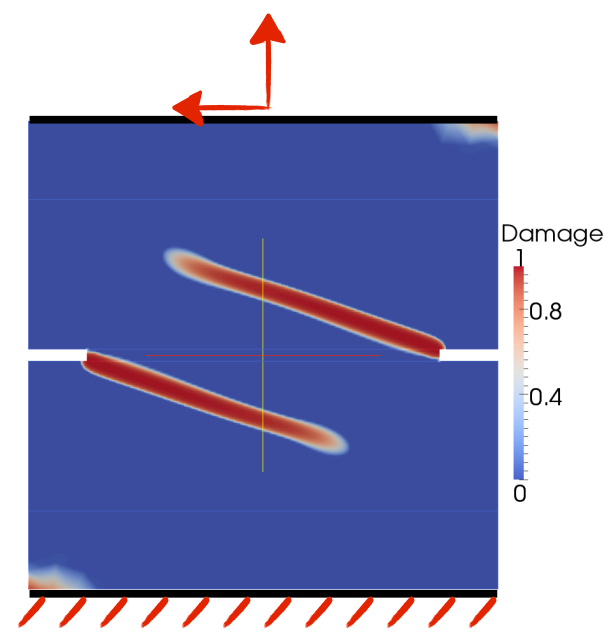

(a)

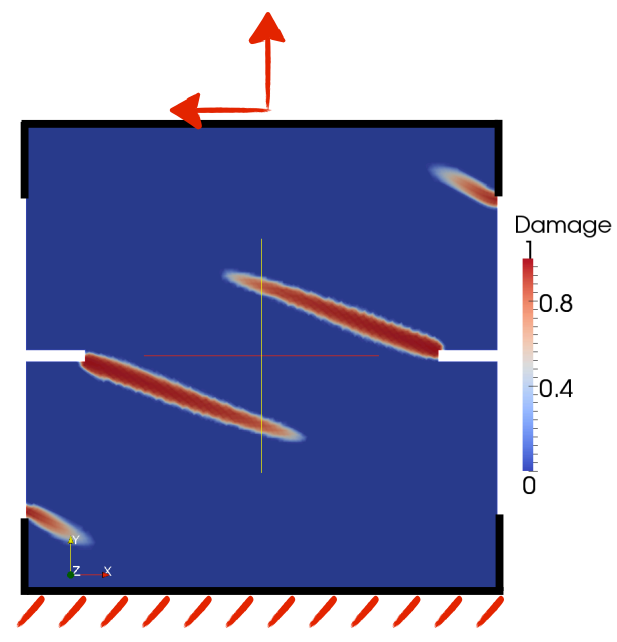

(b)

Figure 5: Comparison of the damage field when flat (a) and U-shaped (b) plates are used for the same prescribed displacements on a double-notched sample. 


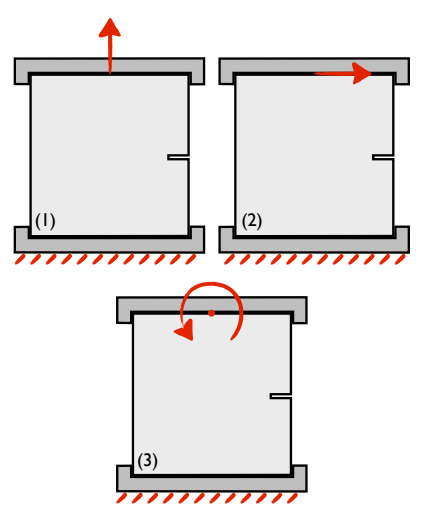

(a)

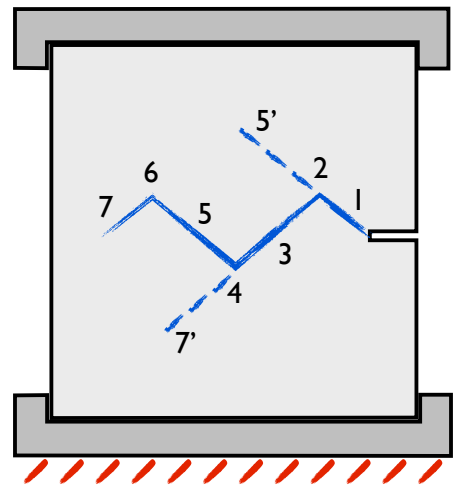

(b)

Figure 6: Three elementary loading paths: 'tension' (a-1), 'shear' (a-2) and 'rotation' (a-3), and the expected crack path (b).

The tensile component is used to initiate and propagate the first crack. The global shear component (to be more accurate, the ratio shear/tension) is used to reorient the crack. Last, the in-plane rotation of the upper plate is combined with shear, which is equivalent to a pure rotation applied to both upper and lower plates in a symmetrical way. It leads to stable crack propagation since it induces a lower tensile stress in front of the crack tip (and possibly compressive stresses) and it also unloads the corners opposite the crack tip that are prone to damage (Figure 7).

The loading path is governed by two main features, namely, the crack is initiated in mixed mode loading and the process zone influence is checked by crack reorientation. The sought crack path (Figure 6(b)) is a first step of initiation and upward propagation (1), then a reorientation downward (2), followed by a subsequent propagation (3), then an upward reorientation (4) with a subsequent propagation (5), and last a reorientation downward (6) with a final propagation (7). The crack will be kept close to the middle of the specimen and will not be too influenced by the imperfections of the boundary conditions. Moreover the shear loading will be applied both ways with crack upward and downward orientations so that crack closure and friction will occur during the steps fol- 


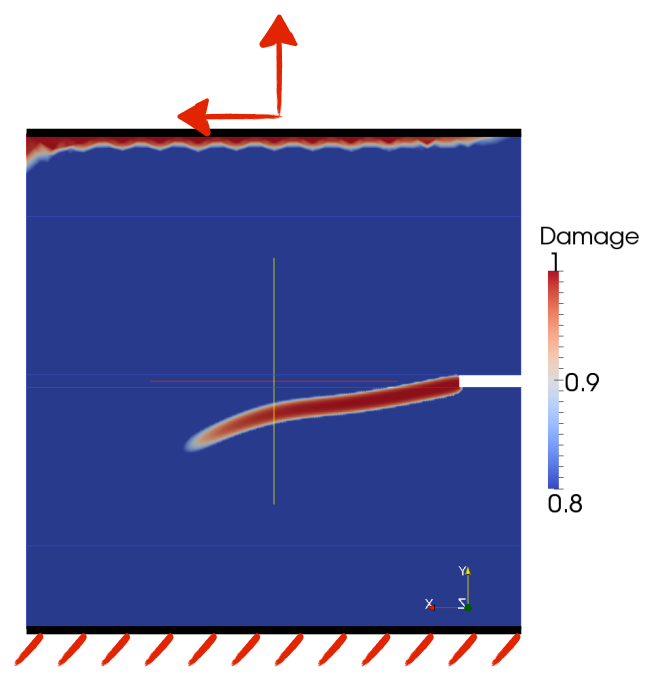

Figure 7: Damage field for a single notched sample subjected to proportional tension-shear.

225 lowing the first propagation. Models taking into account these phenomena can thus be validated, and the validity domain of the models without such features may also be probed. Two propagations are in the upward direction ( $1 \& 5)$, and two in the downward direction $(3 \& 7)$ so that the test is interesting in terms of branching condition at steps (4) and (6). This test addresses in particular the question whether the crack reorients itself and follows the path (5) (resp. path (7)) or branches from the previous reorientation point and follows path (5') (resp. $\left.\left(7^{\prime}\right)\right)$.

\subsection{Instrumentation}

Different kinematic measurement techniques are used to obtain both the boundary conditions and the displacement fields over the sample surface, namely, the hexapod own DIC system, a Linear Variable Differential Transducers (LVDT) setup and several other cameras for additional DIC analyses. Each system has its particular advantages and drawbacks:

- The hexapod own DIC system: fast (i.e., $20 \mathrm{~Hz})$, low uncertainty $(\leq 0.2 \mu \mathrm{m}$ 
tations of the end-effector of the machine are measured.

- The Linear Variable Differential Transducers (LVDT) setup: fast (i.e., $10 \mathrm{~Hz}$ in the present case), low uncertainty ( $\leq 0.1 \mu \mathrm{m}$ each), but only 3 translations and 3 rotations of the end-effector of the machine are obtained through additional calculation.

- Additional DIC systems: slow (i.e., $0.2 \mathrm{~Hz}$ ), higher uncertainty $(2 \mu \mathrm{m})$ but full-field measurements (about $10^{5}$ kinematic degrees of freedom per analyzed image). The measured areas are the specimen surfaces, the Ushaped parts and the reference targets.

The three measurement setups are chosen because their redundancy and complementarity ensure trustworthy results at the micrometer scale. The LVDT and the additional DIC systems will be described in detail in Sections 2.5.1 and 2.5.2 A full description of the hexapod own DIC system can be found in Ref. 45.

\subsubsection{LVDT setup}

An LVDT (WA-T series by HBM) setup composed of 6 sensors is used for measuring the displacements of the end-effector. A rigid steel frame is fitted on the passive hexapod and maintains the sensors as shown in Figure 8(a). Knowing the position of each LVDT and its measurement surface orientation 260 (Figure $8(\mathrm{~b})$ ) enables the 3 relative translations and rotations of the upper end of the hexapod to be calculated. The measurement areas are on the octogonal reference plate (Figure $8(a-b))$, closer to the U-shaped plates than the DIC targets but still a few centimeters away from the specimen upper end. The main interest of this setup is that it is fitted to the machine via a different frame than the DIC control setup, so that spurious displacements due to the applied load deforming the frames would be different and thus detectable.

This setup is sensitive to displacements perpendicular to the axis of each sensor (i.e., bias due to the roughness or surface misalignments). This bias is minimized as much as possible by using flat and ground surfaces, which are 

sensors along these axes. Consequently the deviation of the measurement of each individual sensor is about $1 \mu \mathrm{m}$ and the corresponding standard uncertainty is $0.1 \mu \mathrm{m}$ over a $2 \mathrm{~mm}$ range when only one translation of the end-effector along the sensor axis is considered. Because of the roughness and flatness defects of the surfaces, a set of six linear relationships linking any displacement of the hexapod to those measured by the LVDT setup cannot be stricto sensu considered. Since the first goal of this setup is to check the hexapod displacement during the test, an absolute measurement of the displacement of the end-effector is not necessary per se. Consequently the signal of the sensors are recorded during a displacement without load (i.e., no specimen is mounted), and used as a reference when the signals are measured during the test.

\subsubsection{Additional DIC systems}

Several cameras are placed around the specimen. They are used to measure the displacement of the specimen surfaces, but also the displacement of the U-shaped parts. Since the U-shaped parts and the targets are firmly screwed to the octogonal reference plate that is very stiff, it is considered, as a first approximation, that all these parts have the same rigid body motions, and thus the additional DIC setup provides a redundant information about the endeffector displacements.

Kinematic full fields are measured on each side of the sample with a 'twin' camera setup (i.e., 2 identical cameras $T_{1}$ and $T_{2}$ shown in Figure $9($ a)) and a 2D DIC algorithm using 3-noded triangular elements 48]. The cameras are AVT MANTA G418B (definition: $2048 \times 2048$ pixels, 12-bit digitization), equipped with F1.4 35-mm lenses made by Zeiss. Furthermore, to have redundant measurements and also to quantify out-of plane displacements, global stereo-DIC [49, 50] is performed on each side of the specimen with four cameras $\left(S_{1}^{l}\right.$ and $S_{1}^{r}, S_{2}^{l}$ and $S_{2}^{r}$ shown in Figure $9(\mathrm{a})$ ). These cameras are 60D Digital Single Lens Reflex by Canon, two of them equipped with F2.8 24-mm lenses by Canon, the other two equipped with F2.8 24-mm lenses by Nikon. The lighting 


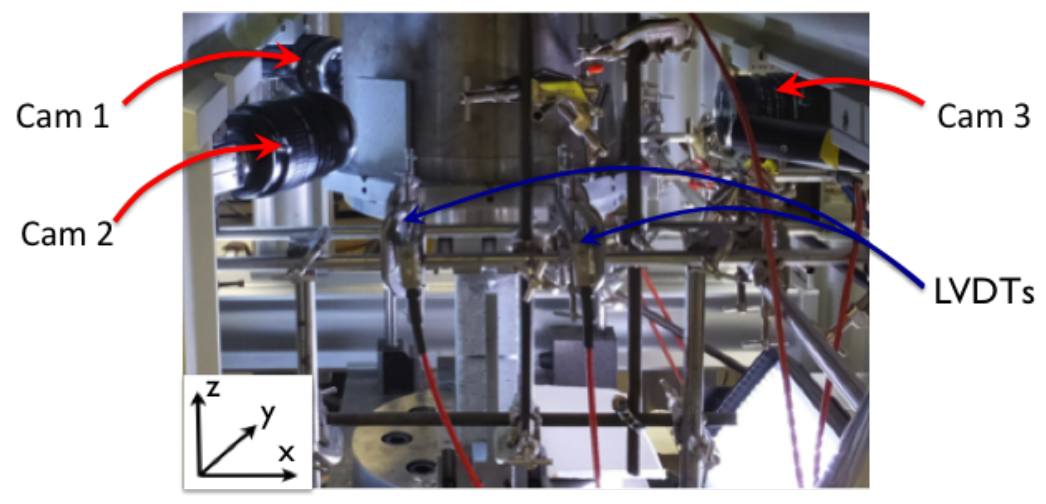

(a)
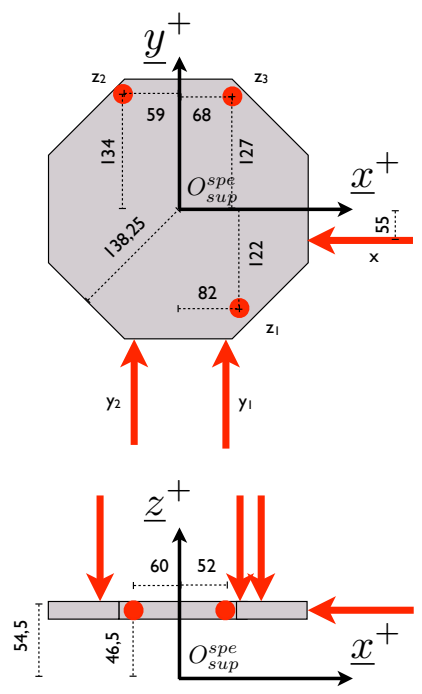

(b)

Figure 8: (a) View of the upper part of setup showing the 3 cameras with their targets and the LVDT setup. (b) Configuration of the LVDT setup (the arrows indicate the measurement location and measurement direction of each LVDT). 
is provided by four LED panels (Microbeam 512 by Flolight) to have a uniform illumination on each side of the specimen.

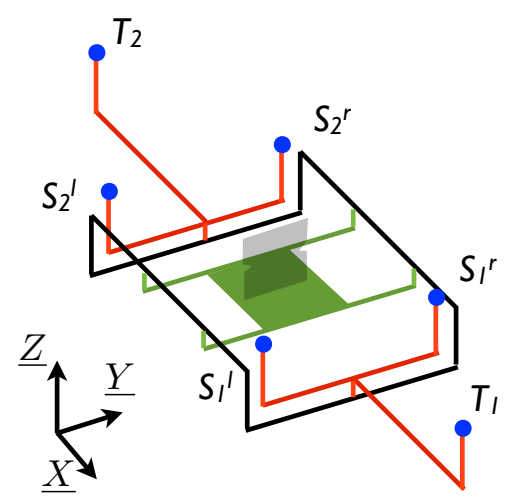

(a)

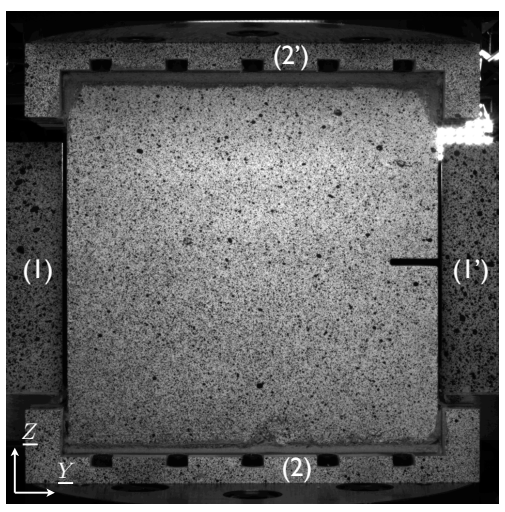

(b)

Figure 9: (a) camera setup and (b) example of image obtained with camera $T_{1}$.

The design of the frame holding all the cameras (those of the hexapod control and the additional ones) is chosen to minimize its sensitivity to the applied load. First, the frame (green part in Figure 9(a)) is linked to the lower U-shaped part through a 45-mm thick steel cylinder and the components of the frame are not subjected to flexural loading during the tests. Second, the frame is fitted through 4 points to the cylinder to be hyperstatic. Last, each set of three cameras on each side of the specimen is mounted on a 'subframe' (red parts in Figure 9(a)). Each subframe is linked to the central frame (black part in Figure $9(\mathrm{a})$ ) by a single connection, so that no forces are transmitted through the subframe besides its own weight. Consequently the relative displacement between the three cameras can only be rigid body motions during the loading of the specimen, except if the subframe itself or the camera own base turn loose.

To be able to correct this last possible effect, and also a possible (and more 315 likely) motion between the two subframes on each side of the specimen, reference targets have been added next to the specimen, fitted to the lower U-shaped part (Figure 9(b)). These 2-mm thick reference targets are covered with a random speckle on each side. They are visible by all 6 cameras so that spurious 
displacements of each camera can be deleted.

These last two points will be detailed.

\subsubsection{Assessment of DIC uncertainties}

The uncertainty of 2D-DIC obtained with cameras $T_{1}$ and $T_{2}$ is assessed. It turns out that air convection effects (shown in Figure 10) have higher contributions in the present case than the uncertainty due to acquisition noise [48] and integer locking [14]. 


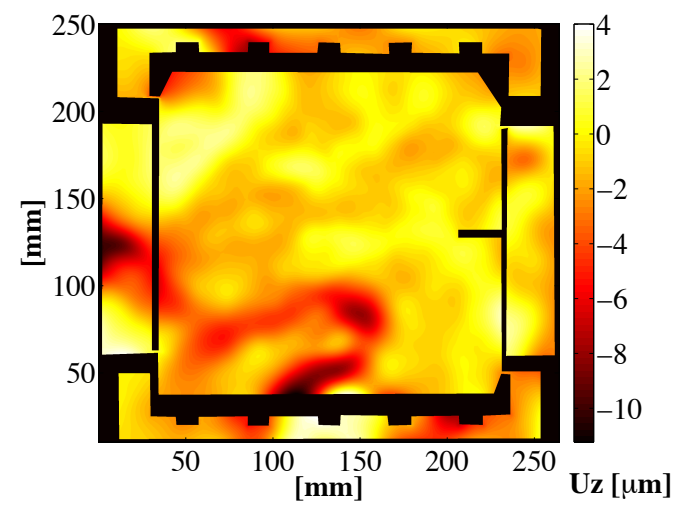

(a)

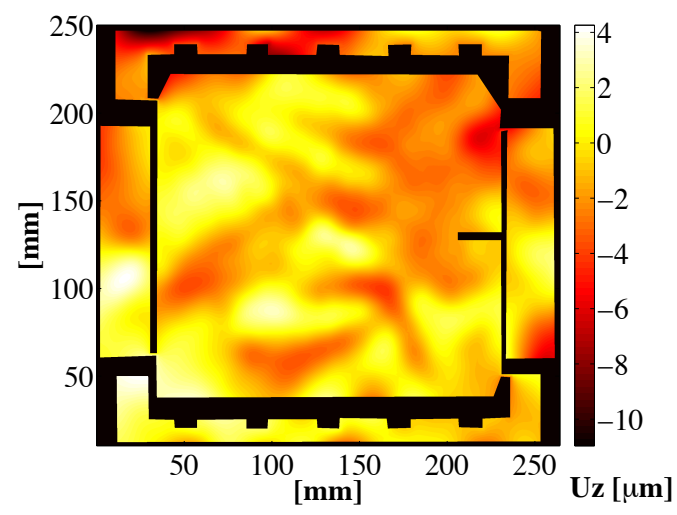

(b)

Figure 10: Example of vertical displacement fields for two consecutive time steps (i.e., $5 \mathrm{~s}$ between (a) and (b)) while the specimen is still. Convection effect is visible. 
A way of reducing this effect during the test is to blow air toward the specimen with a large fan. However the contribution is still larger than integer locking (but small in comparison with the displacement levels of the specimen). The standard displacement uncertainty is about 1.3 (resp. 2.4) $\mu \mathrm{m}$ (i.e., 0.010 (resp. 0.018) pixel along $\underline{Z}$ (resp. $\underline{Y}$ ) direction) with the fan on. This anisotropy may be due to the direction of the air flow. Since a quasi static test is performed, an additional way of lowering the measurement uncertainty due to the high frequency convection phenomenon would be to perform a simple time filtering [51] or to resort to spatio-temporal DIC [52].

The uncertainty of the rigid body displacement between the upper and the lower U-shaped parts obtained by stereo-DIC is assessed with 101 photographs taken when the hexapod stands still. The values are $0.07 \mu \mathrm{m}$ (resp. 0.68, 1.77) for a translation along $\underline{X}($ resp. $\underline{Y}, \underline{Z})$, and $0.0003617 \mathrm{rad}($ resp. 0.0077951, $0.001128)$ for a rotation along $\underline{X}$ (resp. $\underline{Y}, \underline{Z})$.

\subsubsection{Comparison}

360

Figure 11] shows the translations and rotations applied to the specimen without load (before gluing the lower surface for the 'baseline motions' part), or after complete failure (for the 'test motions' part). This second part has been performed after the test to apply exactly the same loading path to check for any difference.

365 The results are given at point $O_{\text {sup }}^{\text {spe }}$ for the three signals, namely, the machine command, the LVDT signals and the DIC data. In the case of DIC, only cameras T1 and T2 are used with 2D-DIC since no out-of plane displacement is applied (i.e., the out-of-plane displacement measured by stereo-DIC is far below the resolution). The presented DIC results are obtained as follow. On each face, the images of camera T1 (resp. T2) are analyzed with the previously mentioned T3-DIC algorithm. For each image (i.e., every $5 \mathrm{~s}$ ), a rigid body motion is fitted on the measured displacement field in the central rectangular region of each U-shaped part. The difference between the motions of the upper and lower U-shaped parts gives the applied in-plane displacement of each side. The mean 


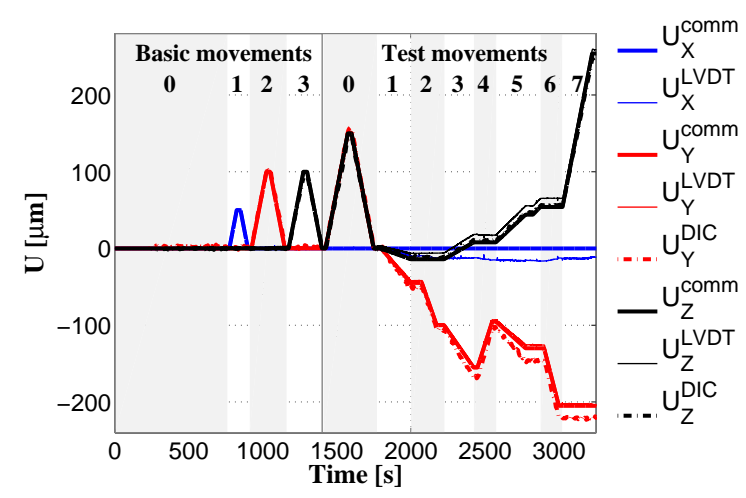

(a)

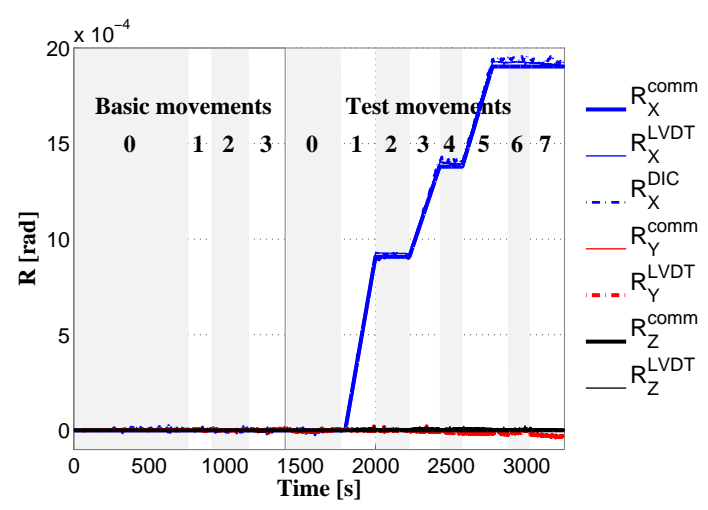

(b)

Figure 11: Translations (a) and rotations (b) expressed at point $O_{\text {sup }}^{\text {spe }}$ during the validation step of the setup without load, prescribed ('comm' subscript), measured by LVDT ('LVDT' subscript) and by DIC ('DIC' subscript). 
375 of the two sides corresponds to the in-plane displacements $U y_{D I C}, U z_{D I C}$, and $R x_{D I C}$ at $U x=0$, which are expressed at point $O_{\text {sup }}^{\text {spe in Figure } 11 .}$

During the steps called 'basic movements', there is first a period where the displacements are recorded while the specimen is held still (step 0), then a $50 \mu \mathrm{m}$ cyclic translation along $X$ is performed(step 1), then a $100 \mu \mathrm{m}$ cyclic translation along $Y$ (step 2) and then along $Z$ (step 3). The displacements corresponding to the test are then performed ('test movements' $0-7$ ), starting with a proportional $150 \mu \mathrm{m}$ loading-unloading $Y-Z$ translation (step 0 ).

The prescribed displacements and the two measurements are in good agreement. During the first translations without rotation, the difference between LVDT measurements and command is less than $2 \%$ of the magnitude of displacement, while the difference between DIC measurements and command is less than $7 \%$ in the worse case (i.e., measured displacement along $Z$ during $Z$ translation). This error has not been explained since the DIC results are trustworthy (i.e., they lead to consistent numerical simulations as shown in Section 3) and the LVDT setup is also very accurate. One must keep in mind that even if this relative error seems large, the corresponding absolute error is very small (few micrometers).

Visible drifts on $R_{X}$ plateau must be considered carefully since these apparent still periods are in fact corresponding to large translations. Consequently these drifts may be interpreted as control errors (i.e., due to the DIC technique used by the control loop), but also as measurement errors of the LVDT setup (Figure 12). During the last plateau for example, the LVDT tips slide over more than $75 \mu \mathrm{m}$ along $Y$ and $200 \mu \mathrm{m}$ along $Z$, while the drift corresponds to a difference of $5 \mu \mathrm{m}$ between the LVDT measurements. Since the linearity error given by the LVDT maker is $1 \mu \mathrm{m}$ for a $2-\mathrm{mm}$ stroke, the drift may come from control errors but also slight flatness defects and misalignments of the octogonal reference part. 




Figure 12: Displacement measurements obtained by the LVDT setup during the validation step.

\section{Test Results}

\subsection{General comments} Moreover the $\underline{X}$ translation is very small : while the biais observed during the displacement without loading (i.e., performed without the specimen, as a reference) is about 12 to $16 \mu \mathrm{m}$, the measure is equal to $\approx 25 \mu \mathrm{m}$ at the 


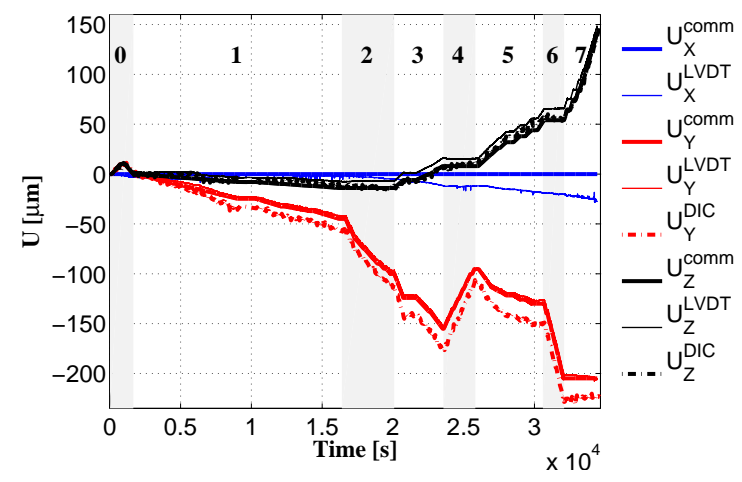

(a)

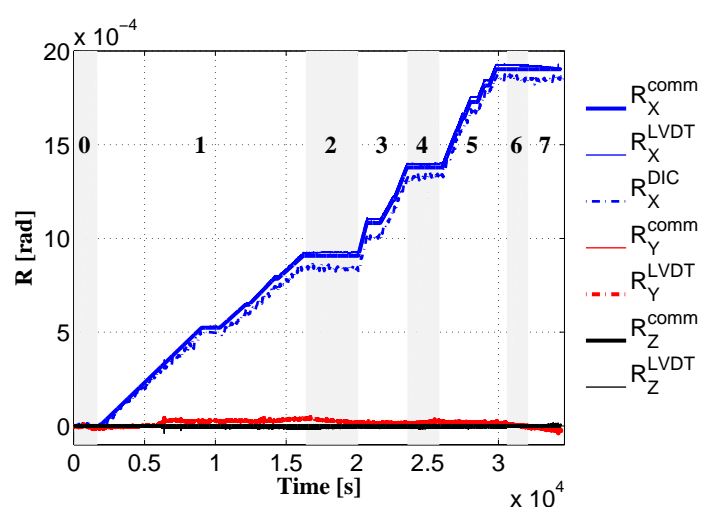

(b)

Figure 13: Translations (a) and rotations (b) expressed at point $O_{\text {sup }}^{\text {spe }}$ during the test: prescribed ('comm' subscript), measured by LVDT ('LVDT' subscript) and by DIC ('DIC' subscript). 
end of the test. This small drift is consistent with the very small out-of-plane translation measured by stereo-DIC (i.e., less than $4 \mu \mathrm{m}$ ). The in-plane feature of the test is also confirmed by the measured forces and torques (Figure 14), namely, $F_{X}, M_{Y}$ and $M_{Z}$ are close to zero.

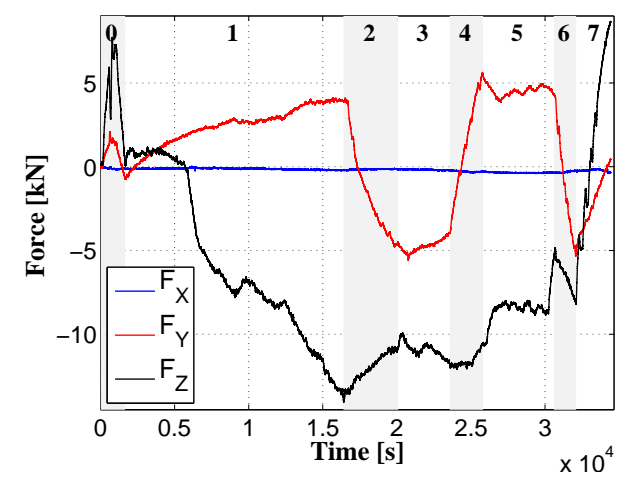

(a)

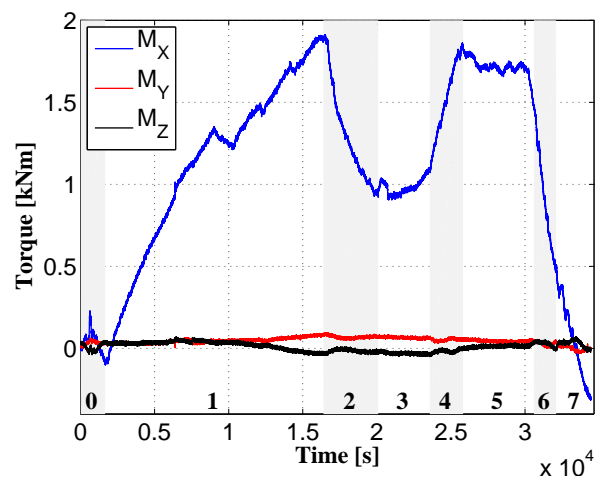

(b)

Figure 14: Forces (a) and Torques (b) measured during the test and expressed at point $O_{\text {sup }}^{\text {spe }}$.

Moreover, the crack propagation is symmetric with respect to the $\underline{Y}-\underline{Z}$ plane as proven by analyzing the displacement fields on both surfaces (Figures 15 and 16) in addition to the final cracked surface (Figure 17). These last observations confirm that the test achieved what was originally planned.

Last, when the final crack is studied with tomography (Figure 18), no par- 

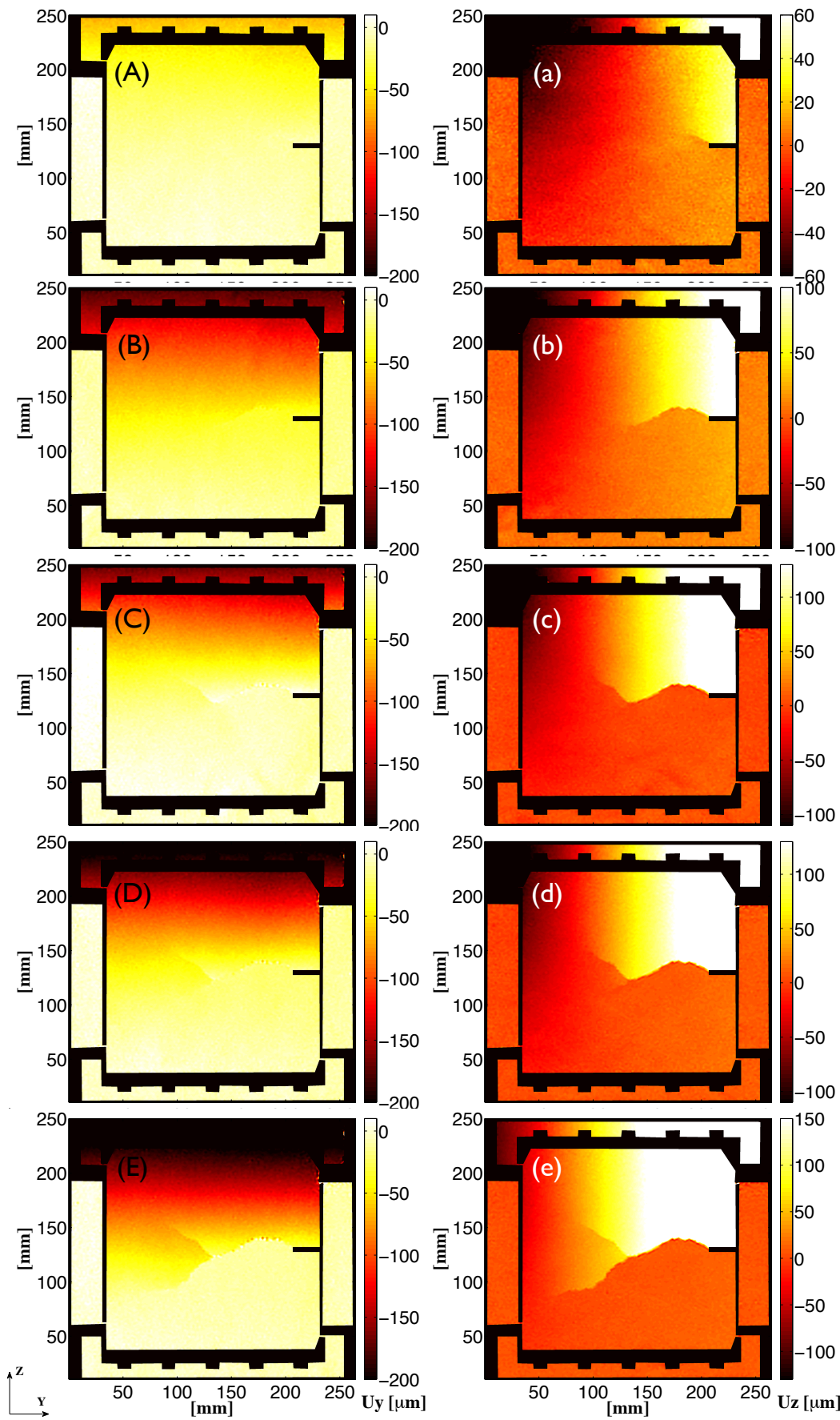

Figure 15: Displacement fields observed on face 1: (A)-(E) show $U_{Y}$ and (a)-(e) $U_{Z}$ components. (A)-(a) (resp. (B)-(b), (C)-(c), (D)-(d), (E)-(e)) correspond to the end of step 1 (resp. step $3,5,6,7)$. 

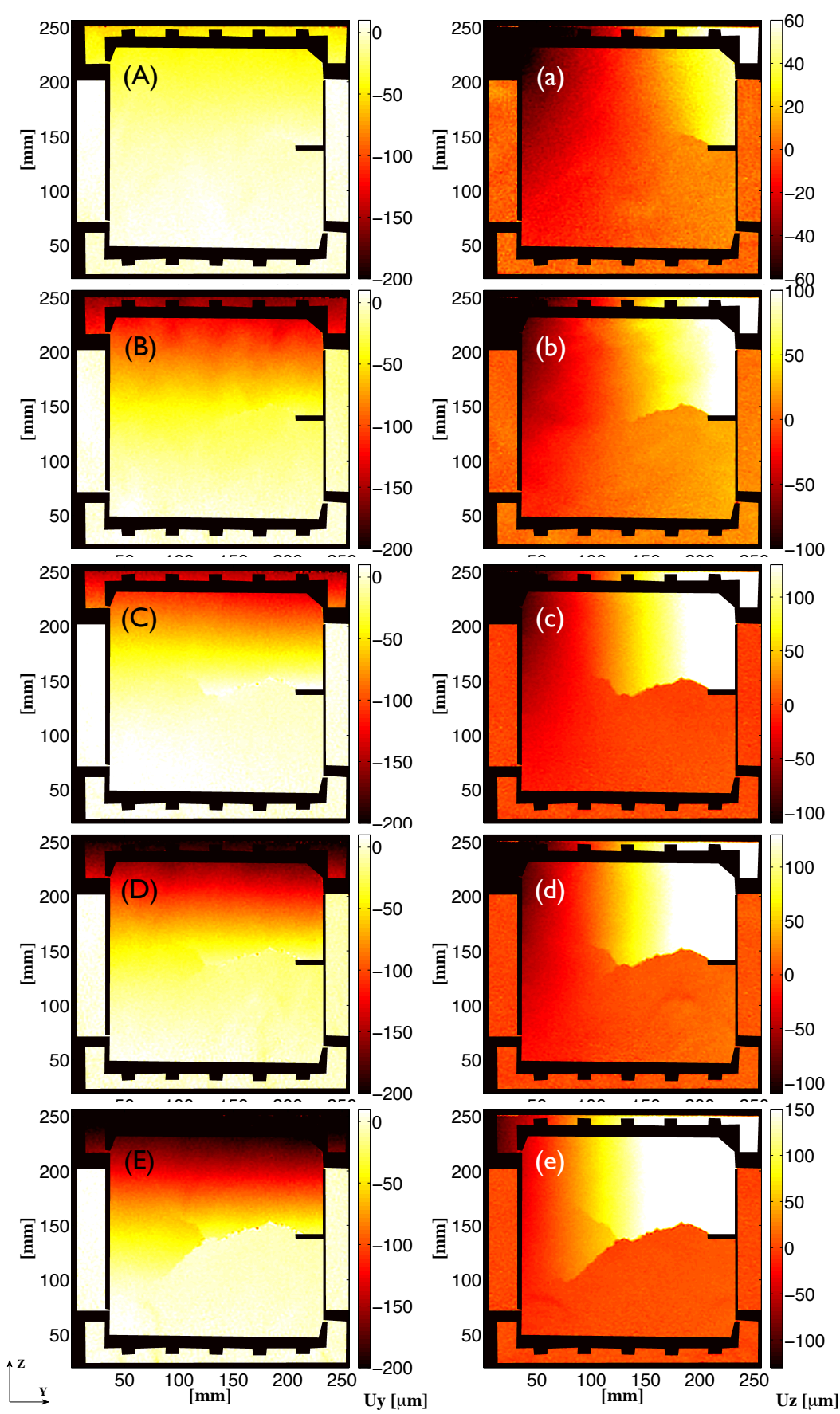

Figure 16: Displacement fields observed on face 2: (A)-(E) show $U_{Y}$ and (a)-(e) $U_{Z}$ components. (A)-(a) (resp. (B)-(b), (C)-(c), (D)-(d), (E)-(e)) correspond to the end of step 1 (resp. step $3,5,6,7)$. Images have been flipped for the sake of comparison with Figure 15 


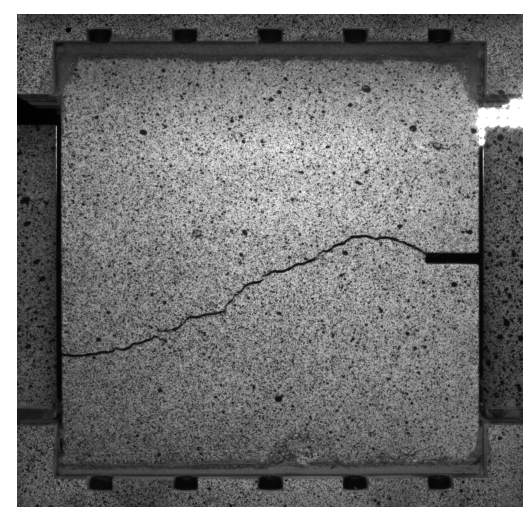

(a)

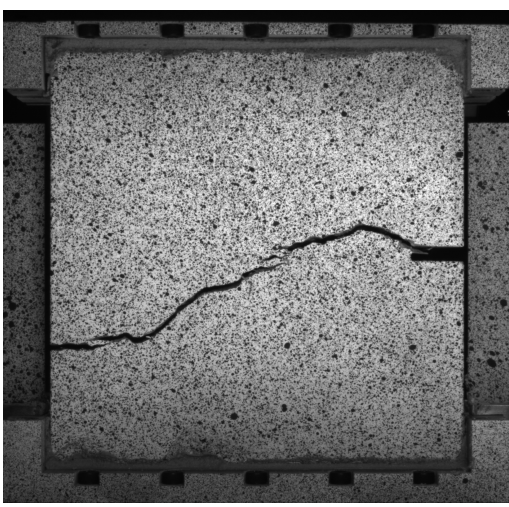

(b)

Figure 17: Specimen after complete crack propagation and the application of an extra translation equal to $\approx 2 \mathrm{~mm}$ along $\underline{Z}$ to enhance the crack visibility: face 1 (a) and 2 (b) (face 2 image is flipped for comparison purposes).

ticular 3D effect is observed, namely, the crack path is nearly the same in every $X$-section. However this result must be taken carefully since the curvature of the crack front along the propagation direction is not detectable with an a posteriori analysis like the present one.

\subsection{Step by step analysis}

Step 0 aims for no initiation, just an elastic loading up to $10 \mu \mathrm{m}$ along $\underline{Y}$ and $\underline{Z}$ followed by an unloading at the same rate. The results show a linear change of the forces and torques with the applied displacements. A sudden drop of $F_{z}$ during the increase of the displacement appears, probably due to slip between two parts of the setup. The goal of this step is apart from checking that every measurement device is working normally to let such type of 'self-positioning' set in before the real loading. No crack is detected by DIC at maximum force nor at the end of the step. One notes a lower stiffness in the $\underline{Y}$ direction in comparison with $\underline{Z}$ in Figure 14 .

Step 1 corresponds to a proportional combination of a translation along $\underline{Y}$ - to induce a global shear stress in the $\underline{Y}-\underline{Z}$ plane - and a translation along $\underline{Z}$ ${ }_{445}$ added to a rotation along $\underline{X}$ - to induce a roughly uniform gradient of tensile 


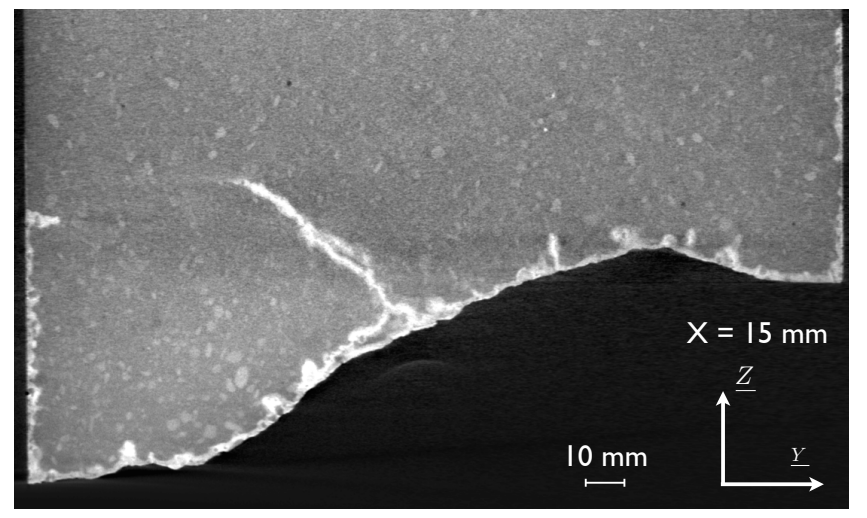

(a)

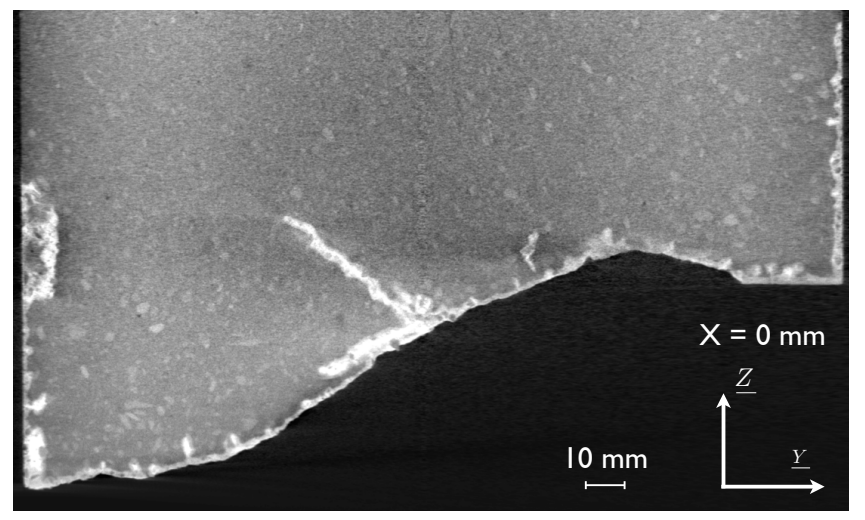

(b)



(c)

Figure 18: Tomographic images of the upper part of the specimen after the test for different $X$ sections. Zinc iodide was used to enhance the crack visibility. 
stresses along $\underline{Z}$. Of course the real local stress field is more complex because of the notch.

At the beginning of the step, $F_{Y}$ and $M_{X}$ are proportional to the applied displacement. The level of $F_{Z}$ remains close to zero since only a gradient of tensile stress is applied with nearly zero mean. At around $t=6000 \mathrm{~s}$, the crack initiates and propagates 'upward' (i.e., toward positive $Z$ values and negative $Y$ values) until the end of the step (see (A)-(a) in Figures 15 and 16). This first propagation is quasistatic : the $F_{Z}$ drop takes about $560 \mathrm{~s}$ so that the control loop has enough time to adapt the actuator lengths (Figure20(b)). In a more general way one notes that the time evolutions of the actuator lengths are smooth, as well as the forces and torques, which confirms the stability of the propagation. Because the section transmitting the $M_{X}$ torque is progressively reduced in the region of tensile stresses (i.e., positive values of $Y$ ), the average force $F_{Z}$ becomes more and more negative with the propagation of the crack. ${ }_{460}$ The variation of $F_{Z}$ corresponds to an indirect sign of crack propagation. The stiffness of the specimen is slightly modified, namely, the slopes of $F_{Y}$ and to a lesser extent of $M_{X}$ are lower than at the beginning of the step.

Step 2 prepares the bifurcation by inverting the sign of $F_{Y}$. As a consequence $M_{X}$ also evolves since the rotation about $\underline{X}$ is not modified during this step. Considering the displacement fields, no visible propagation occurs. The slope of $F_{Y}\left(U_{Y}\right)$ (Figure 19) is equal that of step 0, which means that in spite of the crack opening (up to $\approx 50 \mu \mathrm{m}$ along $\underline{Z}$ near the notch, see Figures 15 (a) and $16(\mathrm{a}))$, the transmission of the load along $\underline{Y}$ through the crack faces is still possible. One does not even see any progressive crack closure during this step for which the slope would evolve.

Step 3 consists of linearly increasing the rotation and the vertical translation to re-propagate the crack. The latter turns and propagates downward (i.e., toward negative $Z$ values) as expected (see Figures 15(B-b) and 16(B-b)). Because of crack propagation, the forces and torques remain constant while the 45 displacement evolves notably.

Step 4 prepares a new bifurcation. Same trends as for step 2 are observed 




(a)

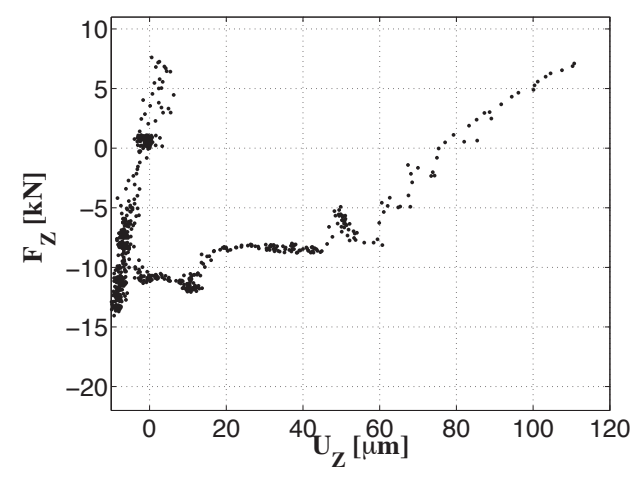

(b)

Figure 19: Evolution of forces versus translation along $\underline{Y}$ (a) and $\underline{Z}$ axes (b). 


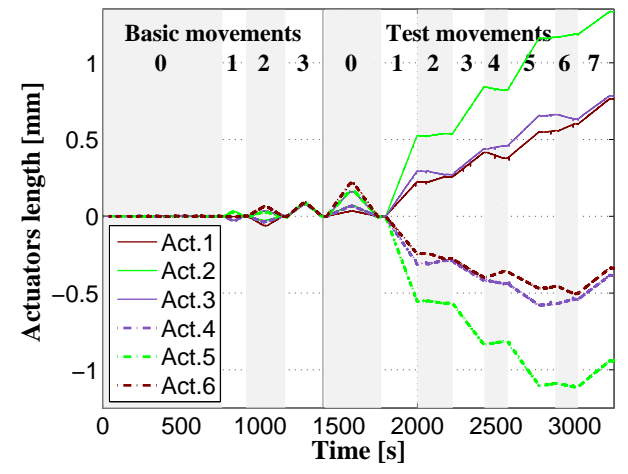

(a)

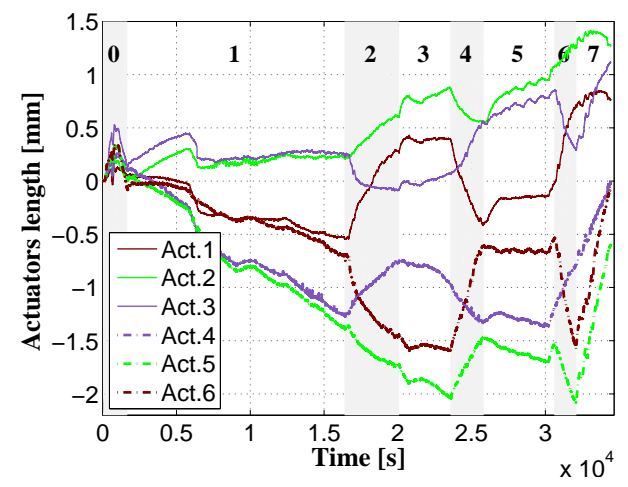

(b)

Figure 20: Actuator length measured during the validation step of the setup (a) and during the test (b). 
except that the change of $F_{Y}$ is inverted (i.e., negative to positive values). The slope of $F_{Y}\left(U_{Y}\right)$ is equal to that of steps 2 and 0 (Figure 19) so that it is concluded that the load along $\underline{Y}$ is still transmitted through the crack faces despite a vertical opening of $\approx 75 \mu \mathrm{m}$ (Figures $15(\mathrm{~b})$ and $16(\mathrm{~b})$ ).

During step 5, the linear increase of rotation and vertical translation induces a bifurcation, so that the crack follows path 5 upward (Figure 6(b)) instead of branching and following path 5'. After a brief decrease of the forces and torques at the beginning of the step, they remain constant or slowly increase during stable crack propagation until the end of the step (Figures 15 (C-c) and 16 (Cc)).

Step 6 prepares another bifurcation (as steps 2 and 4 did). The change of $F_{Y}$ is identical to that of step 2 , namely, from positive to negative values. The $F_{Y}(T y)$ slope is now lower than those of steps 4,2 and 0 . This means that the crack opening (Figures 15(c) and 16(c)) does not enable for the transmission of the load through the complete crack faces. Figures 15(d) and 16.(d) show that the crack has started to branch during this step. This branching induces a small decrease of $F_{Z}$.

During step 7, only a translation along $\underline{Z}$ is applied so that $F_{Z}$ becomes positive. No bifurcation occurs to propagate downward. Instead, branching occurs so that the crack follows path 7' (Figure 6(b)). $F_{Y}$ tends to zero because of the thinning of the ligament and the opening of the crack and thus the larger compliance. The $F_{Z}(T z)$ slope is lower than at step 0 , thereby proving that the load is (nearly) transmitted through the ligament. The crack becomes eventually unstable and complete failure occurs in a dynamic manner, which gives information about the limit of stability.

Last, to check that the whole results are consistent and ready for dataprocessing, a numerical simulation has been performed for each specimen face (since the test is very close to a planar test). A nonlocal scalar damage model [4] 505 without crack closure, briefly summarized in Appendix A, has been used. The boundary conditions are measured on the specimen (not on the U-shape parts) with cameras $T_{1}$ and $T_{2}[53$. The computation converges and the overall trends 
are in good agreement exemplified by the comparison between the simulated and the experimental force-displacement curves shown in Figure 21. The different steps of the test are clearly reproduced and for each one the simulation is consistent.

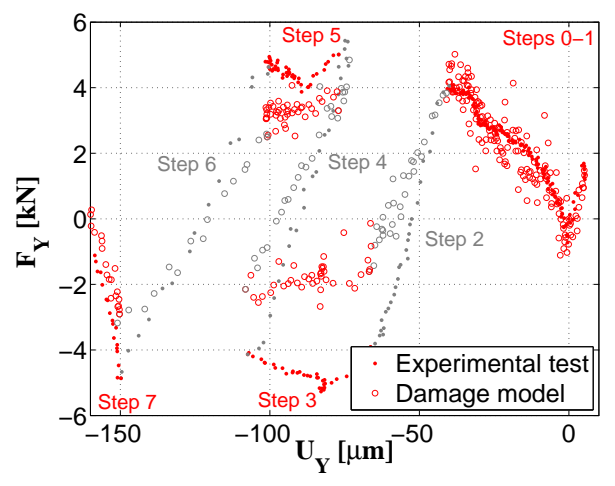

(a)

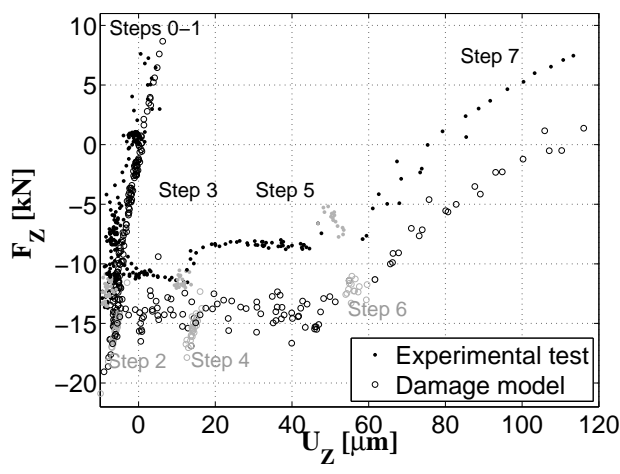

(b)

Figure 21: Comparison between the force-displacement curves predicted by the damage model and the experimental data in shear (a) and tension (b).

The predicted damage field is shown in Figure 22 superimposed with the displacement field at the end of the test. The measured and simulated crack paths are very close. The load and kinematic measurements performed during the test are thus deemed trustworthy. 


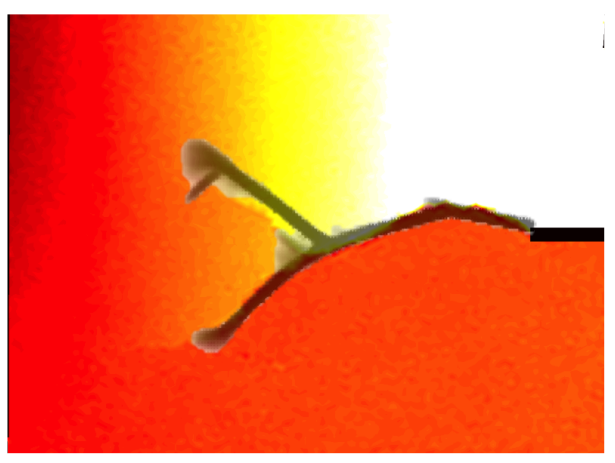

(a)

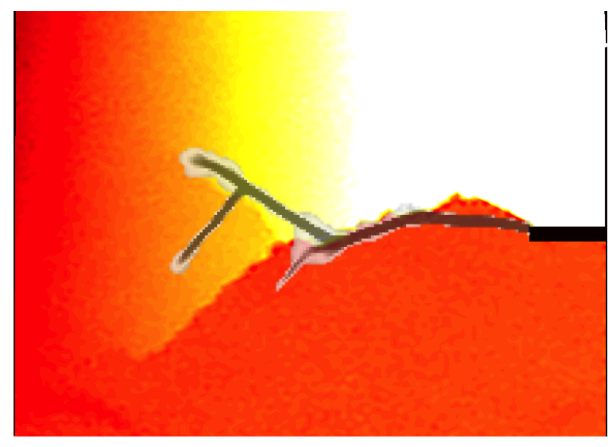

(b)

Figure 22: Comparison between measured vertical displacement field and damage field (thick black line) on face 1 (a) and 2 (b). 


\section{Conclusion}

The goal of the paper is to present a rich and well instrumented case of inplane mixed-mode crack propagation in mortar, which can be used to validate numerical crack propagation / damage models. An innovative 6-axis testing eral full-field kinematic measurement systems. The choice of the loading type, namely, a combination of global tension, shear and in-plane rotation, has lead to reorientations and stable crack propagation phases. The test is deemed rich because it includes several phenomena (i.e., initiation, propagation, bifurcafriction) but not occurring all at the same time. A long step of propagation is obtained between each bifurcation/branching phase, which helps to differentiate the involved phenomena.

It is also considered as well instrumented because a large quantity of data has and torques is of interest to better understand and compare the experimental results to numerical simulations. Tomographic images have also been acquired to check the material heterogeneity and the final 3D crack path.

Several key points will make the numerical reproduction of the test easier.

${ }_{540}$ First, the tested material has been characterized and the main parameters are given. Second, a case with a single crack has been chosen since numerical codes are generally less adapted to reproduce several crack propagations at the same time. Third, full-field measurements have been extensively used to improve the real boundary conditions assessment, and to enable for quantitative 
plane test has been chosen and the static and kinematic measurements prove that the experiment can be considered as in-plane indeed. No tilt of the crack or crack curvature normal to the propagation direction is observed, so that $2 \mathrm{D}$ simulations are also possible.

During the propagation steps, the changes of loading enabled several reorientations of the crack to occur. Bifurcation has been tested three times and the case when the crack can bifurcate or branch to propagate has been tested twice. Bifurcation has been observed for the first step and branching for the second one, which illustrates both cases with an increase of complexity (i.e., a branched crack has to be managed only at the end of the test).

The paper aimed to describe the experimental protocol, the different available data and the general results of the test. It nonetheless does not allow for a fine numerical simulation since the complete experimental data set size is hundreds of megabytes. To use these data and the results of the 3-point flexural tests, readers are welcome to contact the authors or directly download them from ftp.lmt.ens-cachan.fr (anonymous login, then cd toget/ConcreteCrackTests). Several standard specimens (cylinders and bars) are still available to enable for testing not originally foreseen. The presentation of the complete benchmark database (i.e., 11 other tests preformed with the exact same setup) and the preparation of the data for downloading is currently in progress. Since this 'interactive' test leads to a successful stable and zigzagged crack propagation, this work calls for a full automated test (or hybrid test) based on the same principles, testing machine and instrumentation.

\section{Acknowledgements}

This work has been supported and carried out within the EnerCampus framework. It has also benefited from the support of the French 'Agence Nationale de la Recherche' through the 'Investissements d'Avenir' program under the reference ANR-10-EQPX-37 MATMECA. The authors are grateful to B. Smaniotto for the acquisition of tomographic data, and to C. Jailin for assis- 
tance during the test and data-processing.

\section{Appendix A: Damage model}

The nonlocal (gradient) damage model [47] used herein describes the material state by the infinitesimal strain tensor $\underline{\underline{\epsilon}}$ and by a scalar damage field $a$ with $0<a<1$. The stress/strain relationship reads

$$
\underline{\underline{\sigma}}=A(a) \underline{\underline{\underline{\underline{E}}}}: \underline{\underline{\underline{\epsilon}}}
$$

with $\underline{\underline{\underline{\underline{E}}}}$ denotes Hooke's tensor, and $A(a)$ a decreasing function.

The energy function governing the growth of the damage variable is written as

$$
\Phi(\underline{\underline{\epsilon}}, a)=A(a) \Gamma(\underline{\underline{\epsilon}})+k a+\frac{c}{2}(\nabla a)^{2}
$$

where $k a$ stands for the dissipation energy, and $c$ is the nonlocal parameter. These two parameters control both the size of the damage localization zone and

585 the dissipated energy

$$
k=\frac{3 G_{f}}{4 D}, \quad c=\frac{3}{8} D G_{f}
$$

where $G_{f}$ is the fracture energy per unit area, and $D$ the length scale parameter, i.e., half of the damage band width.

The damage criterion is obtained by deriving the function $\Phi$ with respect to the damage variable $a$

$$
f(\underline{\underline{\epsilon}}, a)=-\frac{\Phi}{a}
$$

and the loading-unloading conditions in the Khun-Tucker form are given by

$$
f \leq 0, \quad 0 \leq \dot{a}, \quad \dot{a} f=0 .
$$

The $\Gamma$ function defines the elastic domain shape

$$
\Gamma=\left(\alpha \operatorname{Tr}(\underline{\underline{\epsilon}})+\sqrt{\left.\beta \operatorname{Tr}^{2}(\underline{\underline{\epsilon}})+\gamma \frac{3}{2} \underline{\underline{\epsilon}}^{d e v}: \underline{\underline{\epsilon}}^{d e v}\right)}\right)^{2}
$$

where $\alpha, \beta$ and $\gamma$ are parameters that can be identified from three material properties, namely, the tensile strength, the compressive strength and the shear 
strength. The elastic domain is then an ellipsoid of revolution, differentiating tensile and compressive behaviors.

Last, the loss of material stiffness is expressed as

$$
A(a)=\frac{(1-a)^{2}}{(1-a)^{2}+m a(1+p a)}
$$

where $m$ is a parameter defining the tensile yield stress $\sigma_{y}=\sqrt{\frac{3 E G_{f}}{2 m D}}$ and $p$ the shape parameter for the asymptotic cohesive model.

The parameters used for the reported numerical simulations are given in Table 3

Table 3: Set of material parameters used in the numerical simulations.

\begin{tabular}{|c|c|}
\hline Young's modulus [GPa] & 21 \\
\hline Poisson's ratio & 0.2 \\
\hline Tensile strength $[\mathrm{MPa}]$ & 3.9 \\
\hline Compressive strength $[\mathrm{MPa}]$ & 80 \\
\hline Fracture energy $\left[\mathrm{J} / \mathrm{m}^{2}\right]$ & 100 \\
\hline
\end{tabular}

600

\section{References}

[1] J. Merlet, Parallel robots, Kluwer Academic Publishers, 2000.

[2] V. E. Gough, S. G. Whitehall, Universal tyre test machine, in: Proceedings of the FISITA Ninth International Technical Congress, 1962, pp. 117-137.

[3] D. Stewart, A platform with six degrees of freedom, in: Proceedings of the IMechE, Vol. 180(15), 1965, pp. 371-385.

[4] A. Stokes, M. Gardner-Morse, D. Churchill, J. Laible, Measurement of a spinal motion segment stiffness matrix, Journal of Biomechanics 35 (2002) $517-521$.

610

[5] F. Motoyoshi, M. Takanori, I.Tadashi, K. Takaya, K. Yuichi, I. Satoru, Development of 6 -axis material tester for measuring mechanical spine properties, Journal of Robotics and Mechatronics 18 (2) (2006) 160-166. 
[6] B. Ding, R. M. Stanley, B. S. Cazzolato, J. J. Costi, Real-time FPGA control of a hexapod robot for 6-DOF biomechanical testing, in: IECON 2011 - 37th Annual Conference on IEEE Industrial Electronics Society, 2011, pp. 252-257.

[7] J. Witz, F. Hild, S. Roux, J. Rieunier, Mechanical properties of crimped mineral wools: Identification from digital image correlation, in: Proceedings of the IUTAM Symposium on Mechanical Properties of Cellular Materials, Vol. 12 of IUTAM Bookseries, Springer Netherlands, 2009, pp. 135147.

[8] J. G. Michopoulos, J. C. Hermanson, A. Iliopoulos, Towards a recursive hexapod for the multidimensional mechanical testing of composites, in: Proceedings of the ASME 2010 International Design Engineering Technical Conferences \& Computers and Information in Engineering Conference IDETC/CIE 2010, Montreal, Quebec, Canada., 2010, pp. 1-9.

[9] M. Nierenberger, M. Poncelet, S. Pattofatto, A. Hamouche, B. Raka, J. M. Virely, Multiaxial testing of materials using a stewart platform: Case study of the nooru-mohamed test, Experimental Techniques 38 (2) (2012) 74-83.

[10] M. Sutton, Computer vision-based, noncontacting deformation measurements in mechanics: A generational transformation, Applied Mechanics Reviews 65 (AMR-13-1009, 050802).

[11] P. Burt, C. Yen, X. Xu, Local correlation measures for motion analysis: a comparative study, in: IEEE Conf. on Pattern Recognition and Image Processing, IEEE, New York (NY), USA, 1982, pp. 269-274.

[12] M. Sutton, W. Wolters, W. Peters, W. Ranson, S. McNeill, Determination of displacements using an improved digital correlation method, Image and Vision Computing 1 (3) (1983) 133-139.

[13] Y. Sun, J. Pang, C. Wong, F. Su, Finite-element formulation for a digital image correlation method, Applied Optics 44 (34) (2005) 7357-7363. 
[14] G. Besnard, F. Hild, S. Roux, "Finite-element" displacement fields analysis from digital images: Application to Portevin-Le Châtelier bands, Experimental Mechanics 46 (6) (2006) 789-804.

[15] S. Roux, F. Hild, Stress intensity factor measurements from digital image correlation: Post-processing and integrated approaches, International Journal of Fracture 140 (1-4) (2006) 141-157.

[16] P. Luo, Y. Chao, M. Sutton, W. Peters, Accurate measurement of threedimensional deformations in deformable and rigid bodies using computer vision, Experimental Mechanics 33 (1993) 123-132.

[17] J. Helm, S. McNeill, M. Sutton, Improved three-dimensional image correlation for surface displacement measurement, Optical Engineering 35 (7) (1996) 1911-1920.

[18] B. Bay, T. Smith, D. Fyhrie, M. Saad, Digital volume correlation: threedimensional strain mapping using X-ray tomography, Experimental Mechanics 39 (1999) 217-226.

[19] M. Nooru-Mohamed, Mixed-mode fracture of concrete: an experimental approach, Ph.D. thesis, Technische Universteit Delft. (1992).

[20] M. Nooru-Mohamed, E. Schlangen, J. G. van Mier, Experimental and numerical study on the behavior of concrete subjected to biaxial tension and shear, Advanced Cement Based Materials 1 (1) (1993) 22-37.

[21] J. G. V. Mier, M. Nooru-Mohamed, Geometrical and structural aspects of concrete fracture, Engineering Fracture Mechanics 35 (4-5) (1990) 617-628.

[22] M. Nooru-Mohamed, Mixed-mode fracture of concrete: an experimental approach, Ch. 3.4.2, Ph.D. thesis, Technische Universteit Delft. (1992).

[23] J. E. Jr. Bolander, S. Saito, Fracture analyses using spring networks with random geometry, Engineering Fracture Mechanics 61 ((5-6)) (1998) 569591. 
[24] S. Fichant, C. L. Borderie, G. Pijaudier-Cabot, A comparative study of isotropic and anisotropic descriptions of damage in concrete structures, Vol. 46, Elsevier, 1998, Ch. Damage Mechanics in Engineering Materials, pp. 259-274.

[25] G. Cusatis, Z. Bazant, L. Cedolin, Confinement-shear lattice CSL model for fracture propagation in concrete, Computer Methods in Applied Mechanics and Engineering 195 (52) (2006) 7154-7171.

[26] T. Gasser, G. Holzapfel, 3D crack propagation in unreinforced concrete: A two-step algorithm for tracking 3D crack paths, Computer Methods in Applied Mechanics and Engineering 195 (37-40) (2006) 5198-5219.

[27] G. D. Luzio, A symmetric over-nonlocal microplane model M4 for fracture in concrete, International Journal of Solids and Structures 44 (2007) 44184441.

[28] R. Desmorat, F. Gatuingt, F. Ragueneau, Nonlocal anisotropic damage model and related computational aspects for quasi-brittle materials, Engineering Fracture Mechanics 74 (10) (2007) 1539-1560.

[29] J. F. Unger, S. Eckardt, C. Konke, Modeling of cohesive crack growth in concrete structures with the extended finite element method, Computer Methods in Applied Mechanics and Engineering 196 (41-44) (2007) 40874100.

[30] J. Kozicki, J. Tejchman, Modelling of fracture process in concrete using a novel lattice model, Granular Matter 10 (2008) 377-388.

[31] P. Prochtel, U. Haussler-Combe, On the dissipative zone in anisotropic damage models for concrete, International Journal of Solids and Structures 45 (16) (2008) 4384-4406.

[32] M. Jirasek, P. Grassl, Evaluation of directional mesh bias in concrete fracture simulations using continuum damage models, Engineering Fracture Mechanics 75 (8) (2008) 1921-1943. 
[33] Y. Dong, S. Wu, S. Xu, Y. Zhang, S. Fang, Fracture of concrete structure using simplified meshless method, Cement and Concrete Research 39 (10) (2009) 966-972.

[34] L. N. Lens, E. Bittencourt, V. M. d'Avila, Constitutive models for cohesive zones in mixed-mode fracture of plain concrete, Engineering Fracture Mechanics 76 (14) (2009) 2281-2297.

[35] J. Réthoré, S. Roux, F. Hild, Mixed-mode crack propagation using a hybrid analytical and extended finite element method, Comptes Rendus Mecanique 338 (3) (2010) 121-126.

[36] R. Fenwick, T. Paulay, Mechanisms of shear resistance of concrete beams, Journal of the Structural Division 94 (10) (1968) 2235-2350.

[37] E. Ballatore, A. Carpinteri, G. Ferrara, G. Melchiorri, Mixed mode fracture energy of concrete, Engineering Fracture Mechanics 35 (1-3) (1990) 145157.

[38] D. Brokenshire, Torsional fracture tests, Ph.D. thesis, Cardiff University (1996).

[39] B. Winkler, Traglastuntersuchungen von unbewehrtenund bewehrten Betonstrukturen, Ph.D. thesis, University of Innsbruck (2001).

[40] E. Galenne, B. Masson, A new mock-up for evaluation of the mechanical and leak-tightness behaviour of NPP containment building, in: CCSC, 2012.

[41] AFNOR, Méthodes d'essais des ciments - Partie 1: détermination des résistances mécaniques (NF EN 196-1) (april 2006).

[42] R. TCS, Determination of the fracture energy of mortar and concrete by means of three-point bend tests on notched beams, Materials and Structures 18 (106) (1985) 285-290. 
[43] A. Lopes, F. Almeida, A force-impedance controlled industrial robot using an active robotic auxiliary device, Robotics and Computer-Integrated Manufacturing 24 (2008) 299-309.

[44] D. Wang, R. Fan, W. Chen, Simulation research on adaptive control of a six-degree-of-freedom material-testing machine, International Journal of Advanced Robotic Systems 11 (8) (2014) 1-13.

[45] J. L. Flohic, V. Parpoil, S. Bouissou, M. Poncelet, H. Leclerc, A 3D displacement control by digital image correlation for the multiaxial testing of

$730 \quad$ materials with a Stewart platform, Experimental Mechanics 54 (5) (2014) $817-828$.

[46] F. Hild, S. Roux, Measuring stress intensity factors with a camera: integrated digital image correlation (I-DIC), Comptes-Rendus Mecanique 334 (1) (2006) 8-12.

735 [47] E. Lorentz, S. Andrieux, A variational formulation for nonlocal damage models, International Journal of Plasticity 15 (1999) 119-138.

[48] Z. Tomičević, F. Hild, S. Roux, Mechanics-aided digital image correlation, Journal of Strain Analysis for Engineering Design 48 (2013) 330-343.

[49] B. Beaubier, J. Dufour, F. Hild, S. Roux, S. Lavernhe, K. LavernheTaillard, CAD-based calibration and shape measurement with stereoDIC - principle and application on test and industrial parts, Experimental Mechanics 54 (3) (2014) 329-341.

[50] J.-E. Dufour, B. Beaubier, F. Hild, S. Roux, CAD-based displacement measurements with stereo-DIC. Principle and first validation, Experimental Mechanics.

[51] M. Poncelet, C. Doudard, S. Calloch, F. Hild, B. Weber, Dissipation measurements in steel sheets under cyclic loading by use of infrared microthermography, Strain 42 (2010) 101-116. 
[52] G. Besnard, S. Guerard, S. Roux, F. Hild, A space-time approach in digital image correlation: Movie-DIC, Optics and Lasers in Engineering 49 (2011) $71-81$.

[53] A. Carpiuc, Innovative tests for characterizing mixed-mode fracture of concrete : from pre-defined to interactive and hybrid tests, Theses, Université Paris-Saclay (Nov. 2015). 